\title{
Design and Evaluation of an Optimization Based Approach to Multiple Burst Admission Control for cdma2000 *
}

\author{
Vincent K. N. Lau and Yu-Kwong Kwok \\ Department of Electrical and Electronic Engineering \\ The University of Hong Kong, Pokfulam Road, Hong Kong \\ $\{$ knlau, ykwok\}@eee.hku.hk
}

\begin{abstract}
In our recent study, we have formulated the burst admission control problem for wideband CDMA systems as an integer programming problem. In this paper, we propose and analyze the performance of a novel burst admission technique, called the multiple-burst admission-spatial dimension algorithm (MBA-SD) to judiciously allocate the previous channels in wideband CDMA systems to burst requests. Both the forward link and the reverse link burst requests are considered and the system is simulated by dynamic simulations which takes into account of the user mobility, power control and soft hand-off. We found that significant performance improvement, in terms of data user capacity, coverage, and admission and outage probabilities, could be achieved by our scheme compared to the existing burst assignment algorithms.
\end{abstract}

\section{INTRODUCTION}

Despite the fact that the third generation high speed wireless communication systems are based on the CDMA (code division multiple access) standards $[2,5]$, there are still a number of technical obstacles to be surmounted before such systems can fully achieve the potential. Firstly, spreading limits the permissible data rates in limited wireless spectrum. Yet, the allocated spectrum could be increased in order to support packet data services and this motivates the wideband CDMA systems. Secondly, law of large number does not hold for the relatively small number of packet data users. Therefore, the intrinsic advantage of perfect statistical multiplexing in CDMA systems does not apply to high speed packet data users. In other words, packet data transmissions from data users have to be coordinated carefully and this motivates the need for burst admission control in wideband CDMA systems for packet data users. In fact,

\footnotetext{
*This research was jointly supported by HKU URC seed grants under contract numbers 10203010 and 10203413 , and by a grant from the Hong Kong Research Grants Council under contract number HKU 7024/00E.
}

burst admission control algorithm is very important to the packet data capacity, coverage and the end-to-end delaythroughput performance.

Burst admission control for CDMA systems is a relatively new research topic. Various burst admission control protocols have been proposed recently based on load and interference measurement $[1,3,5,9,10]$. In CDMA systems, the reverse links are interference limited while the forward links are power limited. Thus, for the reverse link, a data burst request could be admitted if the extra interference to the other users is below thresholds. For the forward link, a data burst request could be admitted if the extra loading requirement at the base station is below thresholds. Usually, the burst requests for the forward link and the reverse link directions are asymmetric and are treated independently. In general, the burst admission algorithm could be decomposed into two sub-layers, namely the measurement sub-layer and the scheduling sub-layer [7]. The measurement sub-layer, for example, collects the load and interference measurement of the burst requests from the concerned mobile stations and base stations and evaluate their admission feasibility. The scheduling sub-layer, on the other hand, arbitrates and prioritizes multiple requests based on the current available resources ${ }^{1}$. For every admitted burst request, the output of the burst admission algorithm includes the transmission rate, assigned burst duration and the burst start time. Most existing burst admission control algorithms focus on the measurement sub-layer only. In other words, they are designed to handle single-burst assignment only. For example, in $[3,5]$, only a single data user is considered for the burst admission algorithm. In the cdma2000 system [3, 4], the burst requests are handled on a first-come-first-serve manner. In [9], empirical scheduling such as equal sharing between multiple-burst requests is considered.

In our recent study [7], we have reported a novel formulation of the burst assignment problem as an integer programming problem. In this paper, we propose a novel optimal burst scheduling algorithm to optimize the system throughput and overall packet delay with respect to the data users. For simplicity, we focus on the multiple-burst assignment on the spatial dimension only ${ }^{2}$ Thus, the proposed scheme is called multiple-burst admission-spatial dimension algorithm (MBA-SD), which is described in detail in Section 2. The

${ }^{1}$ In the context of CDMA, system resource refers to the interference or cell loading levels.

${ }^{2}$ The temporal dimension is tackled in another paper [6]. 
performance of the proposed MBA-SD algorithm is evaluated by dynamic simulation [5] which takes into account of the user mobility and soft hand-off (detailed in Section 3). For illustrative purposes, we evaluate the proposed MBA-SD algorithm based on cdma2000 platform although it could be easily applied to other wideband CDMA systems with minor modifications.

\section{MULTIPLE-BURST ADMISSION SPATIAL DIMENSION (MBA-SD) ALGORITHM}

In [7], we have described in detail our integer programming formulation of the multiple-burst admission control problem. In particular, we have defined and derived the objective functions $\mathcal{J}_{1}$ and $\mathcal{J}_{2}$, which take into account:

- system resource utilization, and

- overall system delay.

For details about the formulation, the reader is referred to [7].

\subsection{Optimal Solution}

In this section, we derive the optimal solution to the burst admission problem based on the principle of divide-andconquer. Without loss of generality, we consider the objective function $\mathcal{J}_{2}[7]$ and the forward burst assignment.

Define $\vec{m}$ and $\vec{\mu}$ (column vectors) to be the allocation vector and the system resource vector. The optimization problem to solve is given by:

$$
\begin{aligned}
\mathcal{F}(\vec{\mu}) & =\max _{\vec{m}}\left\{\mathcal{J}_{2}(\vec{m}, \vec{w})\right\} \\
& =\max _{\bar{m}} \sum_{j=1}^{N_{d}}\left(1+\Delta_{j}+\lambda\left(w_{j}\right)^{\beta}\right) m_{j}
\end{aligned}
$$

with the constraint given by the admissible region:

$$
\sum_{j=1}^{N_{d}} a_{k j} m_{j} \leq \mu_{k}, \forall k \in[1, K]
$$

THEOREM 1. The optimization problem (1) could be expressed into the following recursive form:

$$
\mathcal{F}(\vec{\mu})=\max \left\{0, \max _{j: \vec{\mu} \geq \vec{a}_{j}}\left[\left(1+\Delta_{j}+\lambda\left(w_{j}\right)^{\beta}\right)+\mathcal{F}\left(\vec{\mu}-\vec{a}_{j}\right)\right]\right\}
$$

where $\vec{\mu} \geq \vec{a}_{j}$ denotes $\mu_{k} \geq a_{k j} \forall k \in[1, K]$.

Proof. Omitted due to space limitations.

Intuitively, at every step, a unit of resource is allocated to the request contributing the most to the objective function. Since $\vec{\mu}$ is a real vector, the key step in solving the optimization problem (3) is to partition the resource space properly so that $\mathcal{F}(\vec{\mu})$ is constant within each partition. The optimal solution is outlined as follows.
Step I: Resource space partitioning. Define the resource interval $\mathcal{R}_{k}=\left[0,\left(P_{\max }-P_{k}\right)\right]$. The resource space $\mathcal{R}_{+}^{K}$ is given by:

$$
\mathcal{R}_{+}^{K}=\mathcal{R}_{1} \times \mathcal{R}_{2} \times \ldots \mathcal{R}_{K}
$$

Theorem 2. Define the set, $\mathcal{A}_{j}=\left\{\left(\mu_{1}, \ldots, \mu_{K}\right): \mu_{k} \geq\right.$ $\left.a_{k j} \forall k \in[1, K]\right\} \cap \mathcal{R}_{+}^{K}$. The resource space $\mathcal{R}_{+}^{K}$ could $\overline{b e}$ completely partitioned by the set of non-overlapping subspaces, $\mathcal{P}$, given by:

$$
\begin{aligned}
\mathcal{P}= & \left\{\mathcal{S}(0), \mathcal{S}\left(1,\left[j_{1}\right]\right), \mathcal{S}\left(2,\left[j_{1}, j_{2}\right]\right), \ldots, \mathcal{S}\left(N_{d},\left[j_{1}, \ldots, j_{N_{d}}\right]\right):\right. \\
& \left.j_{i 2} \in\left[1, N_{d}\right], j_{1} \neq j_{2} \neq \ldots \neq j_{N_{d}}\right\}
\end{aligned}
$$

where $\mathcal{S}(0)=\mathcal{R}_{+}^{K}-\bigcup_{j=1}^{N_{d}} \mathcal{A}_{j}$ and $\mathcal{S}\left(n,\left[j_{1}, \ldots, j_{n}\right]\right)$ is given by:

$$
\mathcal{S}\left(n,\left[j_{1}, \ldots, j_{n}\right]\right)=\bigcap_{i=1}^{n} \mathcal{A}_{j_{i}}-\bigcup_{j \neq\left[j_{1}, \ldots, j_{x_{1}}\right]} \mathcal{A}_{j}
$$

Note that complete partition implies the following:

$$
\bigcup_{n \in\left[0, N_{d}\right],\left[j_{1}, \ldots, j_{n}\right] \in \mathcal{C}_{n}^{N_{d}}\left(\left[1, \ldots, N_{d}\right]\right)} \mathcal{S}\left(n,\left[j_{1}, \ldots, j_{n}\right]\right)=\mathcal{R}_{+}^{K}
$$

$$
\begin{aligned}
& \mathcal{S}\left(n_{1},\left[j_{1}, \ldots, j_{n_{1}}\right]\right) \bigcap \mathcal{S}\left(n_{2},\left[j_{1}^{\prime}, \ldots, j_{n_{2}}^{\prime}\right]\right)=\Phi \\
& \left.\quad \text { for } \mathcal{S}\left(n_{1},[]\right) \neq \mathcal{S}\left(n_{2},\right]\right)
\end{aligned}
$$

Proof. Omitted due to space limitations.

Note that $\mathcal{S}\left(n,\left[j_{1}, \ldots, j_{n}\right]\right)$ is called the level-n partition.

Step II: Quantization. Since $\vec{\mu}$ is a real vector, we quantize the non-empty partitions of the resource space in order to facilitate the evaluation of $\mathcal{F}(\vec{\mu})$ in these sub-spaces. However, observe that

$$
\mathcal{F}(\vec{\mu} \in \mathcal{S}(0))=0
$$

. Thus, we quantize the level-1 to level- $N_{d}$ nor nempty $_{\text {parti- }}$ tions of the resource space, $\mathcal{S}\left(n,\left[j_{1}, \ldots, j_{n}\right]\right) \neq: \Phi$, only. Define the quantized partition, $\mathcal{Q}\left(n,\left[j_{1}, \ldots, j_{n}\right]\right) \subset \mathcal{S}\left(n,\left[j_{1}, \ldots, j_{n}\right]\right)$, as:

$$
\begin{aligned}
& \mathcal{Q}\left(n,\left[j_{1}, \ldots, j_{n}\right]\right)=\left\{\left(\mu_{1}, \ldots, \mu_{K}\right) \in \mathcal{S}\left(n,\left[j_{1}, \ldots, j_{n}\right]\right)\right. \text { and: } \\
& \mu_{k}=0 \text { if } a_{k j}=0 \forall j \in\left[j_{1}, \ldots, j_{n}\right] \text {, } \\
& \mu_{k}=\max _{j \in\left[j_{1}, \ldots, j_{n}\right]}\left[a_{k j}\right]+i \delta q_{n} \\
& \text { for some } \left.i \in[0,1, \ldots] \text {, if } a_{k j} \neq 0 .\right\}(10)
\end{aligned}
$$

where $\delta q_{n}$ is the quantization interval for level- $n$ partition.

Step III: Partial Resource Allocation. We solve the iterative equation (3) for $\vec{\mu}$ in each element of the quantized partition set $\mathcal{P}$. The sequence of evaluation of $\mathcal{F}(\vec{\mu})$ starts from the zero level partition $\vec{\mu} \in \mathcal{S}(0)$ and then the first level quantized partition $\vec{\mu} \in \mathcal{Q}\left(1,\left[j_{1}\right]\right)$, the second level quantized partition $\vec{\mu} \in \mathcal{Q}\left(2,\left[j_{1}, j_{2}\right]\right)$ and so on because $\mathcal{F}$ 
could be expressed in terms of already found $\mathcal{F}$ at lower level partitions. For any $\vec{\mu} \in \mathcal{Q}\left(n,\left[j_{1}, \ldots, j_{n}\right]\right)$, we have:

$$
\mathcal{F}(\vec{\mu})=\max _{j \in\left[j_{1}, \ldots, j_{n}\right]}\left\{\left(1+\Delta_{j}+\lambda\left(w_{j}\right)^{\beta}\right)+\mathcal{F}\left(-{ }_{n}\left[\vec{\mu}-\vec{a}_{j}\right)\right]\right\}
$$

Note that $\hat{\vec{x}}=-{ }_{n}[\vec{x}]$ is the quantized vector such that:

$$
\begin{aligned}
& |\vec{x}-\hat{\vec{x}}| \leq|\vec{x}-\vec{y}| \\
& \forall \vec{y} \in \mathcal{S}(0) \bigcup_{r=1}^{n} \\
& \bigcup_{\left(p_{1}, \ldots, p_{r}\right) \in \mathcal{Q}_{r}^{n}\left(\left[j_{1}, \ldots, j_{n}\right]\right)} \mathcal{Q}\left(r,\left[p_{1}, \ldots, p_{r}\right]\right)
\end{aligned}
$$

where $\mathcal{C}_{r}^{n}\left(\left[j_{1}, \ldots, j_{n}\right]\right)$ is the combinatorial set given by:

$\mathcal{C}_{r}^{n}\left(\left[j_{1}, \ldots, j_{n}\right]\right)=\left\{\left(p_{1}, \ldots, p_{r}\right): p_{i} \in\left[j_{1}, \ldots, j_{n}\right], p_{i_{1}} \neq p_{i_{2}}\right\}$

Denote the allocation index, $j^{*}$ as $j^{*}=f(\vec{\mu})$ where $j^{*}$ is the result of the max operation in (11). The allocation vector, $g(\vec{\mu})$, is defined as $\left(m_{1}, m_{2}, \ldots, m_{N_{d}}\right)$ such that $m_{j^{*}}=1$ and $m_{j^{\prime}}=0 \forall j^{\prime} \neq j^{*}$.

Step IV: Termination. Define the initial resource vector, $\vec{\mu}_{1}$ as $-N_{d}\left[\left(P_{\max }-P_{1}\right),\left(P_{\max }-P_{2}\right), \ldots,\left(P_{\max }-P_{K}\right)\right]$. ¿From step III, $\mathcal{F}\left(\vec{\mu}_{1}\right)$ has been found with the first allocation index $j_{1}^{*}=f\left(\vec{\mu}_{1}\right)$. The next resource vector, $\vec{\mu}_{2}=$ $\vec{\mu}_{1}-\vec{a}_{j_{1}^{*}}$. Thus, the second allocation index, $j_{2}^{*}=f\left(\vec{\mu}_{2}\right)$. In general, we have the $p$-th resource vector and the $p$-th allocation index given by:

$$
\vec{\mu}_{p}=\vec{\mu}_{p-1}-\vec{a}_{j_{p-1}^{*}}
$$

and

$$
j_{p}^{*}=f\left(\vec{\mu}_{p}\right)
$$

where the iteration stops when $\vec{\mu}_{p_{0}} \in \mathcal{S}(0)$ for some $p_{0}$. The resulting optimal allocation is given by $\sum_{p=1}^{p_{0}} g\left(\vec{\mu}_{p}\right)$.

Note that in the worst case, the total number of partitions in the partition set $\mathcal{P}$ is $2^{N_{d}}$. Thus, the computational complexity of the above algorithm is exponential in $N_{d}$ in the worst case. This motivates the following heuristic approach.

\subsection{Sub-Optimal Solution}

Define the resource index at the $p$-th iteration, $\zeta_{j}^{(p)}$ as:

$$
\zeta_{j}^{(p)}=\min _{k: a_{k j}>0}\left[M_{0}, \frac{P_{m a x}-P_{k}-\sum_{j^{\prime}=1}^{N_{d}} a_{k j^{\prime}} m_{j^{\prime}}^{(p-1)}}{a_{k j}}\right]
$$

where $M_{0}=\min \left[M,\left\lfloor Q_{j_{*}} / T_{l}\right\rfloor\right]$ and $\vec{m}^{(p-1)}=\left[m_{1}^{(p-1)}, \ldots, m_{N_{d}}^{(p-1)}\right.$ is the allocation vector at the $(p-1)$-th iteration. Let $\gamma_{j}^{(p)}$ be the priority function at the $p$-th iteration, which is given by:

$$
\gamma_{j}^{(p)}=\left(1+\Delta_{j}+\lambda\left(w_{j}\right)^{\beta}\right) \zeta_{j}^{(p)}
$$

Intuitively, request that occupies small system resource or request that have a long waiting time should be given higher priority as indicated in (17). The iteration starts at $p=1$ where $\vec{m}^{(0)}=[0, \ldots, 0]$. The algorithm try to allocate $\delta m$ resource to one of the competing requests according to their priorities, $\left[\gamma_{1}^{(p)}, \ldots, \gamma_{N_{d}}^{((p))}\right.$. Suppose $j_{*}^{(p)}$ has the highest priority, then $m_{j_{*}^{(p)}}^{(p)}=\min \left[m_{j_{*}^{(p)}}^{(p-1)}+\delta m, M_{0}\right]$. Note that if
$m_{j_{*}}^{(p)}=M_{0}$, this request is taken out of the competing list in the next iteration. After the allocation, the resource in$\operatorname{dex} \vec{\zeta}$ is updated for the $(p+1)$-th iteration. The iteration stops when there exist some $j$ such that $\zeta_{j}^{(p+1)} \leq 0$.

\section{SIMULATION RESULTS}

The proposed burst admission algorithm is compared with two baseline systems. In baseline $I$, we have the single-burst admission algorithm, which is employed in the cdma2000 system. Specifically, concurrent burst requests are served on a first-come-first-serve basis in cdma2000. In baseline II, we have a multiple-burst admission algorithm but with a herestic scheduling sub-layer, and resource is equally shared between concurrent burst requests. Furthermore, priority is given to the existing burst for new packet arrivals [9].

All the burst admission algorithms are evaluated based on static and dynamic simulations used in [5]. For brevity, we do not describe the simulation details here but refer the readers to [5] for more information about the models. Specifically, we employ static Monte Carlo techniques for generating the coverage results for high speed data users. The cellular architecture is assumed to be a hexagonal grid with base-stations (sectorized with three sectors) located at the center of every hexagon. Distance loss is modeled by $d^{-\delta}$, where $\delta$ is the propagation constant. Shadow fading is modeled using the Mawira model [8] for an urban environment. We repeat the Monte Carlo simulations for 100 times to obtain the static coverage results.

We use a simple model for the mobility of the users. Each user (voice or data) selects a random starting position, which is uniformly distributed over the cell. The direction of motion is also randomly selected. The motion is rectilinear until a call is finished. The speed of motion is assumed to be a constant ( $35 \mathrm{miles} / \mathrm{hr}$ ). Soft hand-off and perfect power control are assumed also. To obtain results of dynamic measurements such as admission and outage probabilities, we performed 10 independent simulations with 5000 calls each.

Figure 1(a) shows the forward link capacity of the three different burst admission control schemes. As can be seen, the proposed MBA-SD algorithm outperforms the two baseline approaches by a considerable margin. This clearly demonstrates that if the extra processing requirements are affordable, optimizing the high data rate bursts scheduling is highly beneficial. On the other hand, as expected, the high capacity is not available throughout the entire cell but just the area near the base-station, as indicated in Figure 1(b).

For the reverse link, we obtain results on admission and outage probabilities, as shown in Figures 2(a) and 2(b). Again, we can see that the MBA-SD approach can allow a high admission probability, through its judicious merging of different requests arrived at different time periods. The MBA-SD approach can also support a much higher number of voice users.

\section{CONCLUSIONS}

We have presented a novel CDMA multiple-burst admission control algorithm, which works by arranging the data bursts judiciously in the spatial dimension such that the optimal al- 


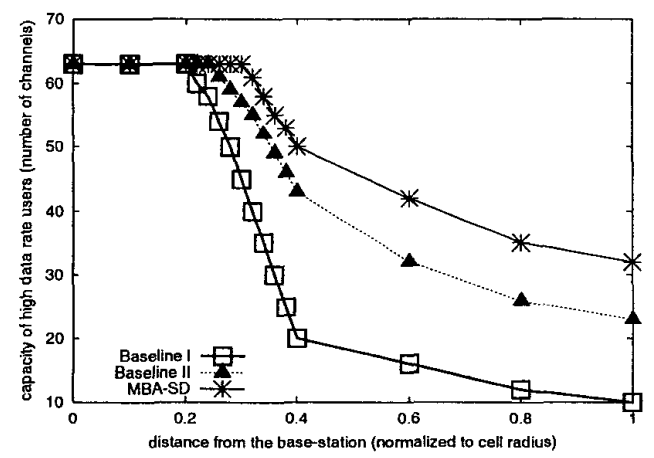

(a) Number of channels available on the forward link with varying distances from the basestation

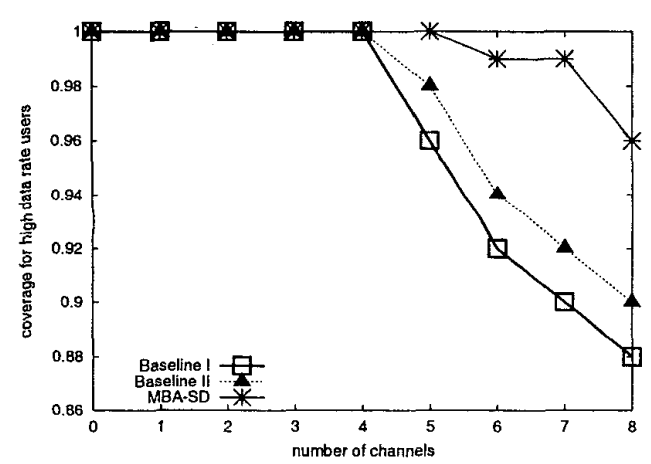

(b) Forward link coverage area as a function of number of channels

Figure 1: Forward link results.

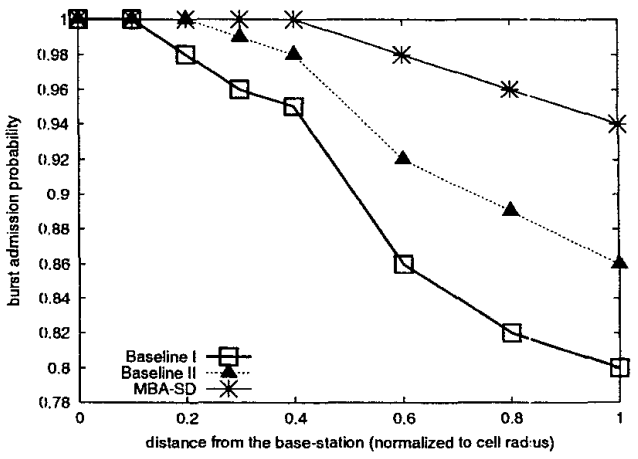

(a) Average admission probabilities on the reverse link

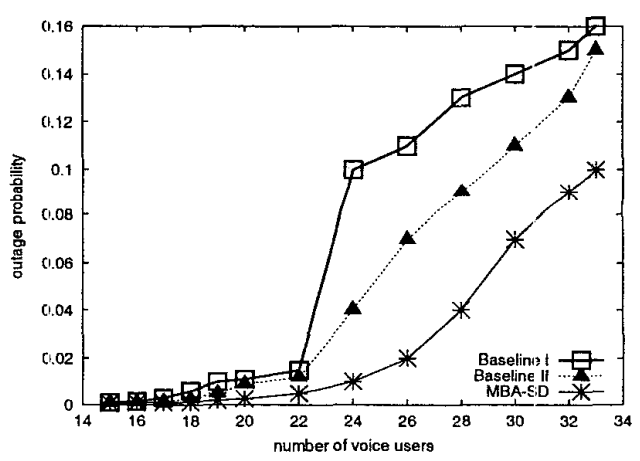

(b) Outage probabilities for voice users

Figure 2: Reverse link results. 
location of channels can be done. Simulation results indicate that the proposed scheme outperforms the first-come-firstserve scheduling scheme and the allocation method used in cdma2000.

\section{REFERENCES}

[1] R. P. Ejzak, D. N. Knisely, S. Kumar, S. Laha, and S. Nanda, "BALI: A Solution for High-Speed CDMA Data," Bell Labs Technical Journal, Summer 1997, pp. 134-151.

[2] N. Guo, S. D. Morgera, and P. Mermelstein, "Common Packet Data Channel (CPDC) for Integrated Wireless DS-CDMA Networks," IEEE Journal on Selected Areas in Communications, vol. 14, no. 4, pp. 735-749, May 1996.

[3] D. N. Knisely, S. Kumar, S. Laha, and S. Nanda, "Evolution of Wireless Data Services: IS-95 to cdma2000," IEEE Communications Magazine, pp. 140-149, Oct. 1998.

[4] D. N. Knisely, Q. Li, and N. S. Ramesh, "cdma2000: A Third-Generation Radio Transmission Technology," Bell Labs Technical Journal, July-Sept. 1998, pp. 63-78.

[5] S. Kumar and S. Nanda, "High Data-Rate Packet Communications for Cellular Networks Using CDMA: Algorithms and Performance," IEEE Journal on Selected Areas in Communications, vol. 17, no. 3, pp. 472-492, Mar. 1999.

[6] Vincent K. N. Lau and Y.-K. Kwok, "Jointly Adaptive High-Data-Rate Burst Admission Control for Wideband CDMA System," submitted for publication.

[7] Vincent K. N. Lau and Y.-K. Kwok, "On Efficient Multiple-Burst Admission Control for cdma2000," Proceedings of the 5th World Multi-Conference on Systemics, Cybernetics, Informatics (SCI'2001), Orlando, Florida, USA, July 2001.

[8] A. Mawira, "Models for the Spatial Correlation Functions of the (log)-normal Component of the Variability of VHF/UHF Field Strength in Urban Environment," Proceedings of the 3rd IEEE Int'l Conf. Personal, Indoor, and Mobile Radio Communications, pp. 436-440, Oct. 1992.

[9] S. Nanda, K. Balachandran, and S. Kumar, "Adaptation Techniques in Wireless Packet Data Services," IEEE Communications Magazine, pp. 54-64, Jan. 2000.

[10] A. Sampath and J. M. Holtzman, "Access Control of Data in Integrated Voice/Data CDMA Systems: Benefits and Tradeoffs," IEEE Journal on Selected Areas in Communications, vol. 15, no. 8, pp. 1511-1526, Oct. 1997. 